\title{
Epidural electric stimulation of posterior structures of the human lumbar spinal cord: 1. muscle twitches - a functional method to define the site of stimulation
}

\author{
M Murg ${ }^{1}, \mathrm{H}_{\text {Binder }}{ }^{1,2}$ and MR Dimitrijevic*,2,3 \\ ${ }^{1}$ Maria-Theresien-Schloessel Neurological Hospital, Vienna, Austria; ${ }^{2}$ Ludwig Boltzmann Institute for Restorative \\ Neurology and Neuromodulation, Vienna, Austria; ${ }^{3}$ Department of Physical Medicine and Rehabilitation, Baylor \\ College of Medicine, Houston, Texas, USA
}

\begin{abstract}
Objectives: To describe an electrophysiological method for determining the relation between lumbar cord dorsal roots and cathode of epidural electrode for spinal cord stimulation (SCS). Materials and methods: Data has been collected from 13 subjects who have been under evaluation of effectiveness of SCS for control of spasticity. Induced muscle twitches from both quadriceps $(\mathrm{Q})$, adductors $(\mathrm{A})$, hamstrings $(\mathrm{H})$, tibial anterior muscles $(\mathrm{TA})$ and triceps surae muscles (TS) were simultaneously recorded with surface-electrode polyelectromyography (pEMG) and analyzed for amplitudes, latency times and recruitment order.

Results: Stimulation of dorsal lumbar cord structures evoked characteristic EMG events during muscle twitch responses. Their amplitudes varied with stimulus strength. Latency times were rather invariable regardless of stimulus strength. Two distinct recruitment orders were demonstrated depending on whether the stimulating cathode was placed over the upper (= response from quadriceps and/or adductor muscles) or the lower (= response from tibialis anterior and triceps surae) lumbar cord segments. The chances to stimulate upper lumbar cord segments are best around the 12th thoracic vertebra.

Conclusions: pEMG recording of muscle twitches enables us to accurately differentiate between upper and lower lumbar cord segments. Furthermore, our findings regarding amplitude, latency and recruitment order strongly suggest that we stimulate posterior roots not posterior columns of the lumbar spinal cord.
\end{abstract}

Spinal Cord (2000) 38, 394-402

Keywords: spinal cord stimulation; placement of electrode; lumbar spinal cord

\section{Introduction}

Spinal cord stimulation (SCS) is a widely used method to control pain, movement disorders, spasticity and chronic limb ischemia. In all these treatment protocols, the clinical outcome hinges on patient selection and optimal placement of the stimulating electrode in the epidural space. ${ }^{1-3}$ Although the technique of placing the epidural electrode is rather simple, difficulties do arise when the surgeon tries to target a specific portion of the spinal cord.

In the past decade, significant progress has been made in defining both structural (ie radiological) and functional (ie paresthetic) criteria to define the location of an SCS electrode relative to specific spinal cord structures. ${ }^{4,5}$ This does not apply, however, in patients with impaired sensory functions of the spinal cord. This means that there is a need for neurophysiological assessment procedures based on muscle twitches that

*Correspondence: MR Dimitrijevic, Ludwig Boltzmann Institute for Restorative Neurology and Neuromodulation, Hofzeile 18-20, A1190 Vienna, Austria are independent of paresthetic sensations. ${ }^{6,7}$ Furthermore, a better understanding of the neurophysiology of spinal cord circuitry and its involvement in sensorymotor functions in completely or partially isolated spinal cord from the brain ${ }^{8,9}$ has opened new perspectives regarding the control of spasticity and neuroaugmentation of residual motor control. ${ }^{10}$ Since this circuitry is confined to specific spinal cord regions, it becomes relevant that we also learn to identify these spinal cord structures when stimulating patients who lack sensory functions.

\section{Materials and methods}

\section{Patients}

Thirteen patients, who were referred to our clinical program of restorative neurology for treatment of spasticity, were included in the study. Evaluating the effect of SCS on spasticity gave us an opportunity to collect data on muscle twitch patterns related to the 
various positions of the stimulating electrode. The study was approved by the local ethics committee, and all patients gave their informed consent.

Included were able-bodied patients who had suffered from SCI involving spasticity (Ashworth $3.3 \pm 0.6 \mathrm{SD}$; range $2.3-4.0)^{\mathrm{T1}}$ for at least 1 year. Excluded were patients with ongoing infection or neurological complications of the primary disease.

The mean age in this series was $28.8 \pm 10.8(18-57)$ years. Eight of the patients were men, five were women. A mean of $44.5 \pm 26.3(16-97)$ months had elapsed since the respective injuries. Eight patients had cervical SCI, five patients had thoracic lesions. Six patients were classified as Frankel A, four patients as Frankel B, three patients as Frankel C. ${ }^{12}$ The range of ASIA motor scores was 3-89 $(45.5 \pm 24.4)$, the range of sensory ASIA scores was $23-108(63.5 \pm 23.2)$ for pinprick and 23-108 $(64.6 \pm 23.4)$ for light touch. ${ }^{13}$ Pertinent clinical data are summarized in Table 1.

\section{Surgical procedure}

The surgical procedure of placing the electrode was carried out by a team comprising a neurosurgeon/ orthopedic surgeon, a neurologist/clinical neurophysiologist and a nurse qualified in technological support. A quadripolar electrode (3487A, Medtronic) was placed in local anesthesia percutaneously in the posterior epidural space at vertebral levels Th11 to L1. The position of the electrode was confirmed by fluoroscopy. The four contacts of the quadripolar electrode were labeled $0,1,2$ and 3 (most rostal $=0$, most caudal $=3$ ). The electrode was placed over the posterior median aspect of the lumbar cord and connected to an external stimulator (Model 3625, Medtronic). Intra-operative stimuli were applied at a pulse width of $210 \mu$ s and a repetition rate of $5 \mathrm{~Hz}$. Stimuli were intensified in 0.5 Volt increments $(0-10 \mathrm{~V})$

The final position of the electrode was determined based in the recruitment order of muscle twitches induced by electric stimulation in the upper segments of the lumbar cord. It was assumed that either contact 0 or 1 was located over the L2 cord segment. After an appropriate test period using an open system with an external stimulator, the electrode was finally connected to the implanted pulse generator (Itrel 3, Model 7425, Medtronic) to form a fully integrated closed system.

\section{Recording of muscle twitches}

Beckman recessed silver-silver surface electrodes were bilaterally (3 $\mathrm{cm}$ apart) placed over the bellies of quadriceps $(\mathrm{Q})$, adductor $(\mathrm{A})$, hamstring $(\mathrm{H})$, tibial anterior (TA) and triceps surae (TS) muscles. The skin was slightly abraded so that an electrode impedance of less than $5 \mathrm{kOhms}$ was reached to reduced or eliminate artifacts. Electromyographic (EMG) activity was recorded with Grass 12A5 amplifiers (Grass Instruments, Quincy, MA, USA) with a gain of 5000 over a bandwidth of $50-800 \mathrm{~Hz}(-3 \mathrm{~dB})$, digitized at 1800 samples per second per channel at a bit depth of 12 bits using a Codas ADC system (DATQ Instruments, Akron, OH, USA). All recordings were conducted with the subject in a comfortable supine position. Stimuli were applied at a pulse width of $210 \mu \mathrm{s}$ and low frequencies (open system: $5 \mathrm{~Hz}$; closed system: $2.1 \mathrm{~Hz}$ ) in $1-$ Volt increments $(0-10 \mathrm{~V})$. The threshold is the stimulus of lowest amplitude which can induce EMG response $(1-4,5 \mathrm{~V})$. EMG responses of quadriceps or/ and adductor were expected when the cathode was over the L2 cord segment, additional hamstring responses were expected over the L3 segment. Tibial anterior responses were expected over the L4-L5

Table 1 Summary of clinical data of studied patients

\begin{tabular}{|c|c|c|c|c|c|c|c|c|c|}
\hline No & Sex & Age & $\begin{array}{l}\text { Implant } \\
\text { after SCI } \\
\text { (months) }\end{array}$ & $\begin{array}{l}\text { Level of } \\
\text { the lesion }\end{array}$ & Frankel & $\begin{array}{l}\text { ASIA } \\
\text { motor }\end{array}$ & $\begin{array}{l}\text { Sensibel } \\
\text { pinprick }\end{array}$ & $\begin{array}{l}\text { IA Sensibel } \\
\text { light touch }\end{array}$ & $\begin{array}{c}\text { Ashworth } \\
\text { LE }\end{array}$ \\
\hline 1 & M & 25 & 34 & $\mathrm{C} 4$ & A & 3 & 28 & 28 & 3.2 \\
\hline 2 & $\mathrm{M}$ & 22 & 61 & C6 & A & 16 & 23 & 23 & 2.4 \\
\hline 3 & $\mathrm{~F}$ & 25 & 35 & D6 & $\mathrm{C}$ & 67 & 87 & 87 & 3.0 \\
\hline 4 & M & 33 & 22 & $\mathrm{C} 7$ & B & 27 & 74 & 74 & 3.6 \\
\hline 5 & M & 18 & 35 & $\mathrm{C} 4$ & A & 16 & 62 & 62 & 3.2 \\
\hline 6 & $\mathrm{~F}$ & 33 & 38 & D5 & A & 50 & 79 & 79 & 3.8 \\
\hline 7 & $\mathrm{~F}$ & 22 & 72 & D2 & B & 50 & 60 & 70 & 2.5 \\
\hline 8 & M & 21 & 16 & D10 & B & 50 & 64 & 64 & 2.3 \\
\hline 9 & $\mathrm{~F}$ & 18 & 22 & D4 & A & 50 & 50 & 44 & 3.6 \\
\hline 10 & $\mathrm{~F}$ & 33 & 31 & D5 & A & 50 & 50 & 54 & 4.0 \\
\hline 11 & M & 57 & 28 & $\mathrm{C} 8$ & $\mathrm{C}$ & 89 & 60 & 66 & 3.5 \\
\hline 12 & M & 40 & 88 & C6 & $\mathrm{C}$ & 74 & 108 & 108 & 3.5 \\
\hline 13 & M & 28 & 97 & C6 & B & 49 & 81 & 81 & 4.0 \\
\hline Mean & & 28.8 & 44.5 & & & 45.5 & 63.5 & 64.6 & 3.3 \\
\hline SD & & \pm 10.8 & \pm 26.3 & & & \pm 24.4 & \pm 23.2 & \pm 23.4 & \pm 0.6 \\
\hline Range & & $18-57$ & $16-97$ & & & $3-89$ & $23-108$ & $23-108$ & $2.3-4.0$ \\
\hline
\end{tabular}

SCI, Spinal Cord Injury; LE, lower extremity 
segments, triceps surae responses over the L5-S1 segments. For maximum responses, we applied stimuli of up to $10 \mathrm{~V}$, however for stimulation of the dorsal roots at the threshold level we used stimulus strength not higher than $4,5 \mathrm{~V}$. All data were stored and analyzed off-line. Electromyographic recording and setting for epidural stimulation is shown in Figure 1.

Data analysis

The recorded EMG potentials of muscle twitches were analyzed using CODAS ADC (DATQ-Instruments Akron, OH, USA) software. Latency times were measured manually and are given as milliseconds between onset of the stimulus and first deflection of evoked EMG responses. Amplitudes were measured from the peak of the largest positive potential to the peak of the largest negative potential. Latencies and amplitudes were calculated at threshold and maximum stimuli based on three single muscle twitches obtained with different polarities $(0-/ 3+$ and $0+/ 3-)$.

\section{Results}

In the first part we shall present EMG characteristics of elicited muscle twitches, and in the second part we

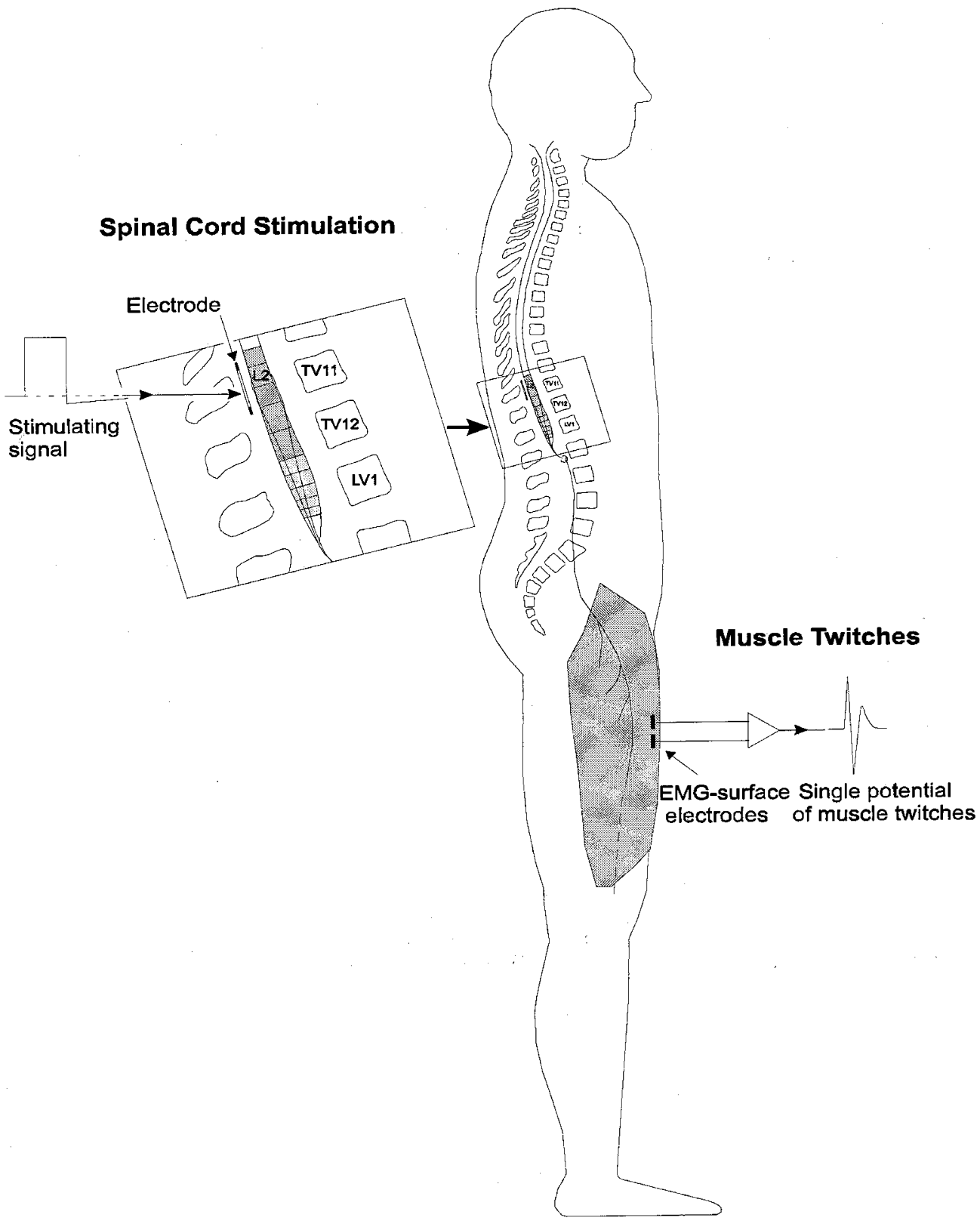

Figure 1 Surface-electromyography of muscle twitch and setting of epidural stimulation 
shall describe the recruitment order of muscle twitch responses during stimulation of selected posterior lumbar cord structures.

EMG-characteristics of muscle twitch response potentials Stimulation of dorsal structures resulted in biphasic, triphasic or even polyphasic potentials. The characteristic single EMG muscle twitch responses at threshold and maximum level are shown in Figure 2A,B,C. Amplitudes obtained at threshold stimuli varied according to muscle groups and the site of stimulation. At maximum stimuli the smallest amplitudes $(1.03 \pm 0.85 \mathrm{mV})$ were obtained with tibial anterior, the largest amplitudes $(2.19 \pm 0.96 \mathrm{mV})$ with hamstring muscles (Figure 2D).

Despite these considerably different amplitudes obtained for the various muscle groups and stimulus levels, the latency of responses was essentially constant (Figure 3A,B). The mean latency at threshold stimuli was $10.1 \pm 1.8 \mathrm{~ms}$ for the three thigh muscles and $16.0 \pm 2.3 \mathrm{~ms}$ for the leg muscles (Figure 3C). At maximum stimuli it was $9.5 \pm 1.2 \mathrm{~ms}$ for thigh muscles and $17.0 \pm 1.8 \mathrm{~ms}$ for leg muscles (Figure 3D). These rather constant and short latencies are suggestive of monosynaptic responses elicited by stimulation of large primary afferents of dorsal roots (dorsal root reflexes), which are within half time of tendon reflex latencies of the studied muscle groups.

All things considered, we may conclude that by stimulating dorsal roots along the L2 to L5 cord segments, it is possible to elicit corresponding muscle twitches. The amplitudes of these muscle twitches is quite variable depending on stimulus strength, whereas the latency times of the response are interindividually constant and regardless of stimulus strength.
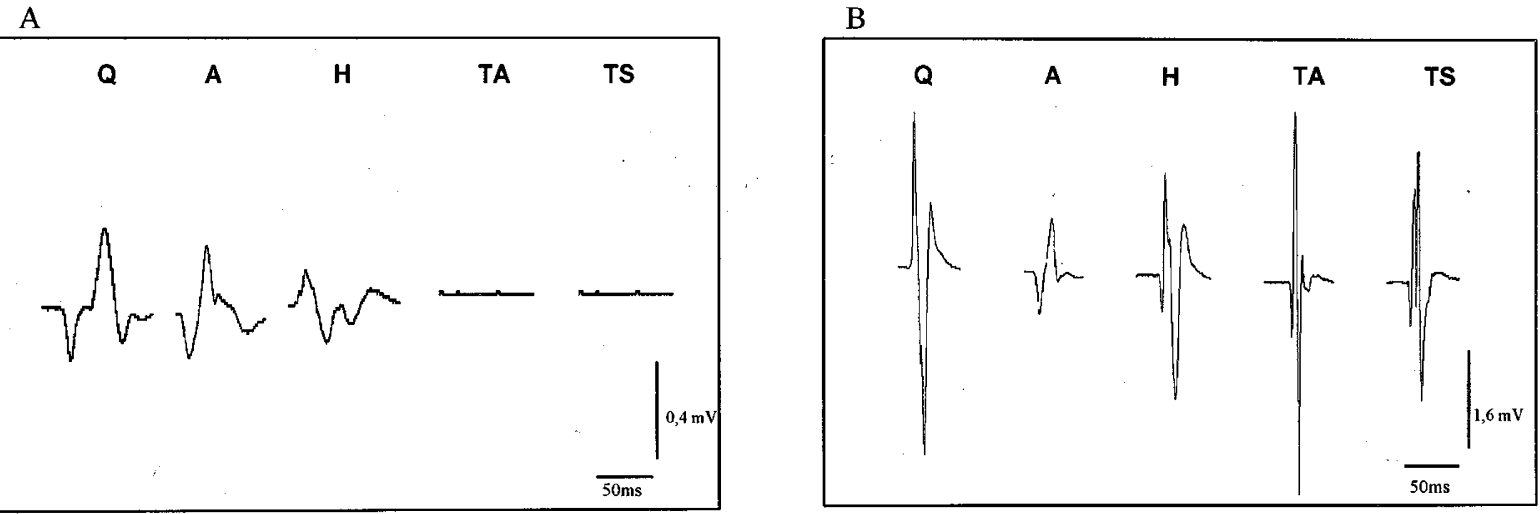

$\mathrm{C}$

D
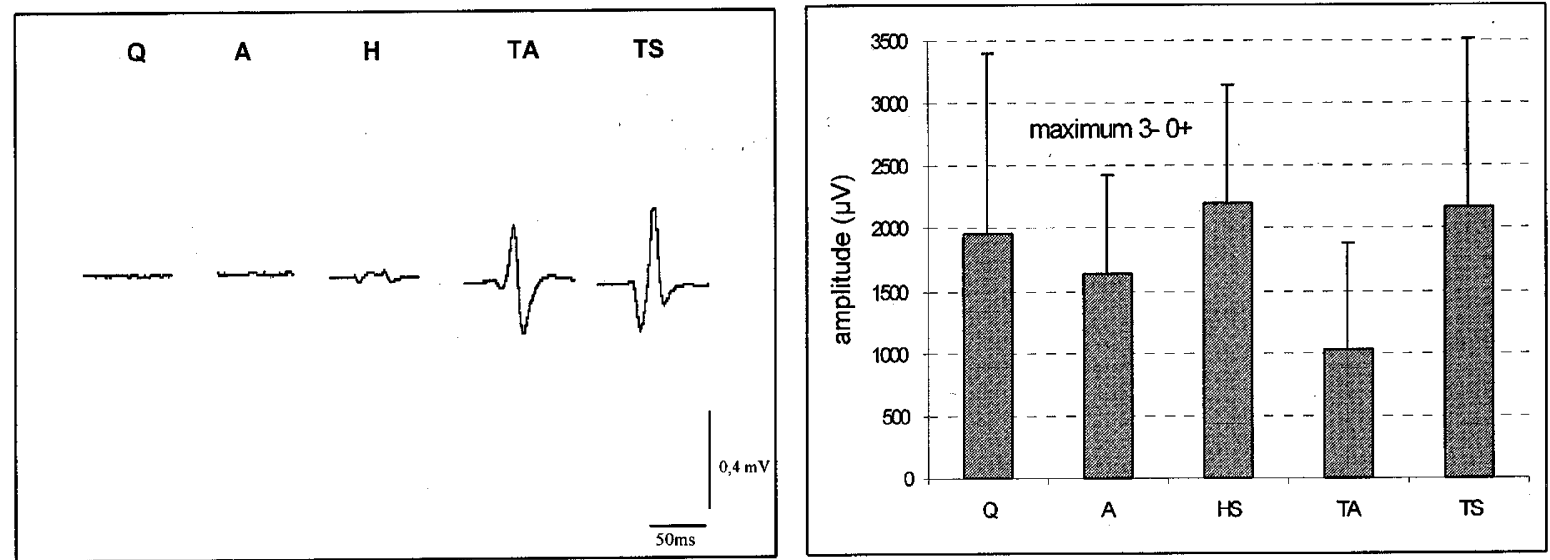

Figure 2 Single potentials of EMG responses of muscle twitches of Quadriceps (Q), Adductor (A) and Hamstrings (H) obtained from a single subject (A) and of Tibial anterior (TA) and Triceps surae (TS) from another single subject (C) at the threshold level. Single potentials of EMG responses of all muscle groups at the maximum level of stimulation (B). The histogram shows the mean amplitudes of EMG responses of muscle twitches $( \pm \mathrm{SD})$ obtained at maximum (D) levels of stimulation for various muscle groups in all 13 patients 
A

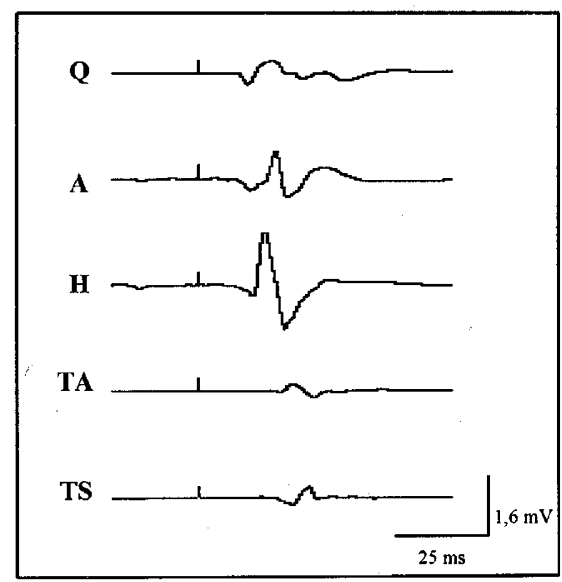

$\mathrm{C}$

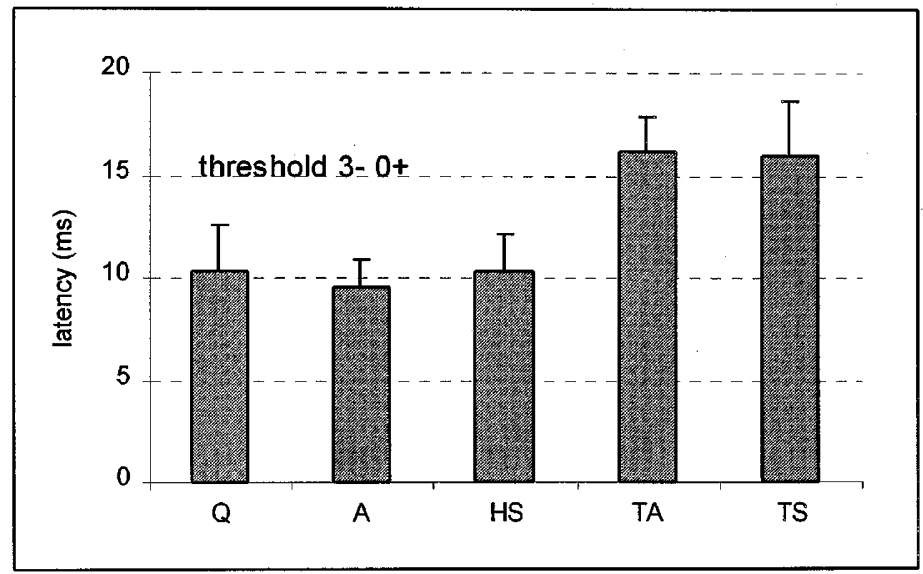

B

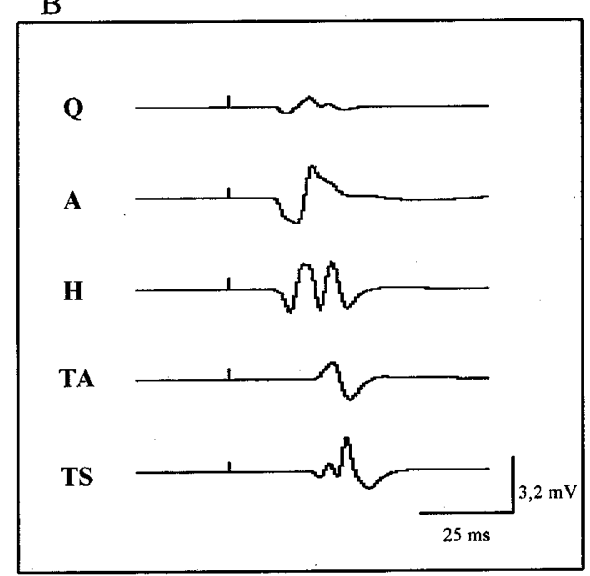

D

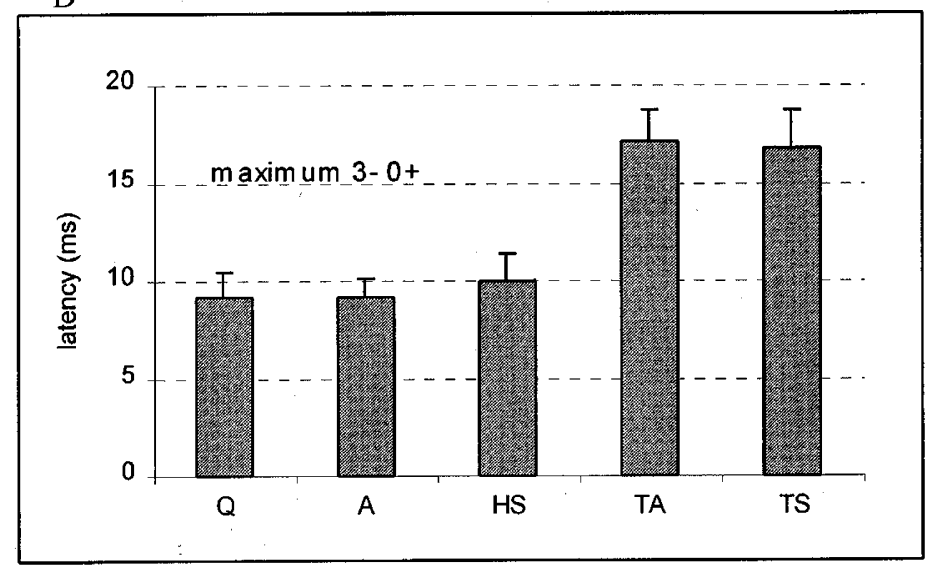

Figure 3 Latency times of single EMG potentials of different muscle groups elicited by threshold stimulus (A) and by maximal stimulus (B) on a single subject (No. 7) and mean latency times \pm SD of all 13 patients at threshold (C) and maximum (D) stimulus levels

\section{Recruitment order of muscle twitch responses}

Our approach to controlling spasticity involved a variety of electrode positions along vertebrae TV 11 to LV 1 that gave us an opportunity to study their effect on muscle twitch recruitment patterns. An illustrative example obtained in one representative subject is displayed in Figure 4. As we obtain marked responses in quadriceps/adductor, minimal responses in hamstring at the stimulus strength of $4 \mathrm{~V}$, and no responses in tibial anterior and triceps surae, we may assume that the stimulating cathode is located above the second lumbar cord segment, which is where dorsal spinal roots enter the spinal cord (Figure 4A). In this particular case the X-ray showed that the tip of the electrode (contact 0) was located just below the upper surface of the body of the 12th thoracic vertebra. The recruitment order changed quite dramatically on moving the electrode under fluoroscopic visualization caudally to the upper surface of the first lumbar vertebra. In that position, the $3 \mathrm{~V}$ threshold stimulus elicited the largest amplitudes in triceps surae and tibial anterior, moderate amplitudes in hamstring and adductor, and minimal amplitudes in quadriceps. Judging from this recruitment order we assume that the cathode is located between the fourth and fifth lumbar cord segments (Figure 4B). Thus the recruitment order and amplitude of muscle twitches depend on the site of stimulation of the lumbar spinal cord.

Table 2 summarized the relationship between the fluoroscopically verified location of the stimulating cathode and the corresponding muscle recorded in our series of 13 subjects. These data indicate that Xray studies of the thoracic-lumbar spine are not useful in defining particular sites of stimulation. They only help us to locate the cathode relative to the spine, but the position of the stimulating electrode relative to specific spinal cord segments can only be identified by the EMG muscle twitch 
A
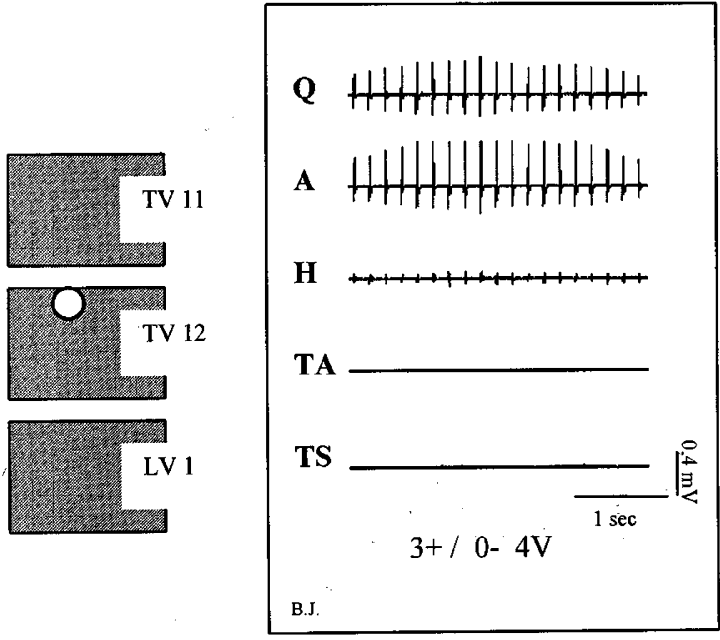

B

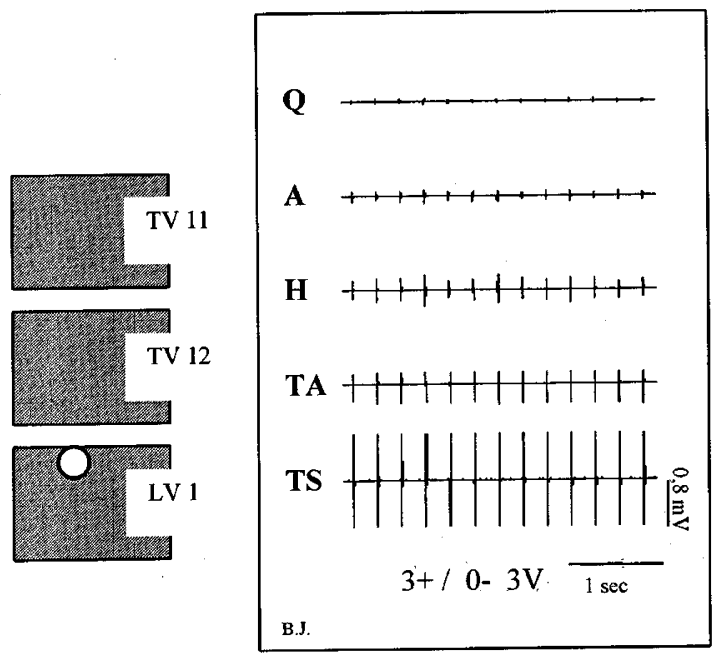

Figure 4 Recruitment order of EMG responses of muscle twitches in a single subject (No. 1): (A) when the cathode is placed over the second lumbar spinal cord segment, or just below the upper surface of the 12th thoracic vertebra; (B) when the cathode is placed over the fourth lumbar spinal cord segment, or below the upper surface of first lumbar vertebra

responses patterns. Bearing in mind that the upper and lower lumbar cord segments may be located anywhere between the 11 th and 12th thoracic and first lumbar vertebrae, the chances to elicit muscle twitches corresponding to the upper lumbar segments are better when the cathode is at the level of the 12th thoracic vertebra. Likewise, EMG of muscle twitches corresponding to the lower lumbar segments are more likely evoked with the cathode over the first lumbar vertebra.

\section{Discussion}

In the study, we have demonstrated that the threshold stimulus $(1-4,5 \mathrm{~V})$ of an epidural electrode placed over the medial posterior portion of lumbar cord segments will induce bilateral muscle twitch responses. When the cathode is placed closely over dorsal root nerve fibers of the second lumbar cord segment, then a relatively small stimulus will elicit twitches of both the quadriceps and adductor muscles. When the cathode is placed closely over the dorsal root fibers of the fourth and fifth lumbar cord segments, the same type of threshold stimulus will elicit muscle twitches of tibial anterior and triceps surae.

Anatomical conditions in that part of the spinal cord favor dorsal root stimulation and minimize the possibility if inadvertently stimulating dorsal column fibers, which are a much more complex structure. Dorsal root fibers can be compared to simple straight nerves. Their myelinated fibers have a diameter of $10-$ $20 \mu \mathrm{m}$, whereas posterior column fibers only have a diameter of $5-6 \mu \mathrm{m} .{ }^{14}$ Therefore the threshold stimulus for dorsal roots is less than half the level required for dorsal columns. In addition to the greater fiber diameter, there are other factors contributing to the lower stimulation threshold in dorsal root fibers: (i) they have a different orientation relative to the electrode; (ii) they are curved while dorsal column fibers are straight; and (iii) they travel across the interface between a low-conductivity (spinal cord) and a high-conductivity (CSF) compartment. ${ }^{15-17}$

A significant difference between the stimulation thresholds in dorsal root fibers as compared to posterior column fibers becomes apparent when we place the electrode over the lower thoracic cord segments. At threshold stimuli we record lower abdominal muscle twitches corresponding to dorsal roots. By intensifying the stimulus up to $10 \mathrm{~V}$ we elicit muscle twitches of the upper (quadriceps, adductor) but not of the lower (tibial anterior, triceps surae) lumbar segments. This finding adds to the evidence that we did not stimulate posterior columns in our study.

The origin of the dorsal afferent rootlets is in cell bodies of the dorsal root ganglion, which is located outside of the spinal cord. ${ }^{18}$ A number of spinal fibers attached to a shallow depression in the posterolateral sulcus fuse to form a dorsal nerve root structure. ${ }^{19,20}$ In a similar way, small fibers fuse along the anterior aspect of the spinal cord to form a ventral root. Before they form the nerve root, however, the sensory and motor fibers are distinct entities to lateral sac. ${ }^{21}$ Due to this anatomical feature, both fiber types have different stimulation thresholds. With the cathode placed over the medial part of posterior lumbar spinal cord structures, the threshold is lower for sensory than for motor fibers because they are closer to the source of stimulation.

From a neurophysiological standpoint, we would expect to see shorter latencies in ventral than in dorsal 
Table 2 The position of the cathode of electrode according to the persistent polarity is presented in relation to the spine vertebra 12th thoracic- and first lumbar vertebra (one exception of 11th thoracic vertebra) as well as to the corresponding muscle twitches amplitudes in average to the segments of lumbar spinal cord

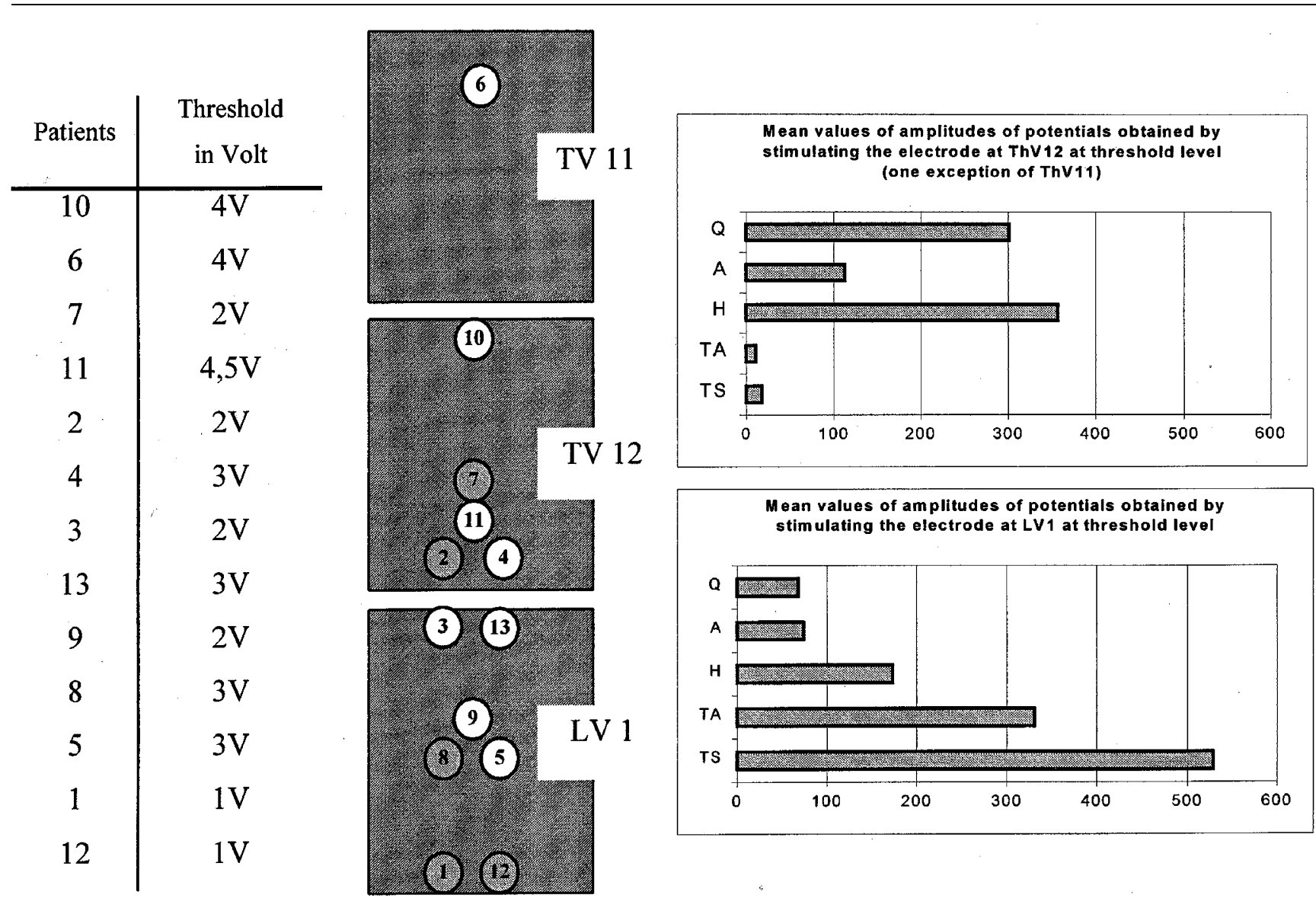

$\mathrm{TV}=$ thoracic vertebra; $\mathrm{LV}=$ lumbar vertebra; $\mathrm{V}=$ Volt. Numbers of patients are rowed according to the position of cathode from rostral to caudal; $\bigcirc$ stimulating cathode of these positions muscle twitches of L2 and L3 segments were recorded; stimulating cathode of these positions muscle twitches of L4 and L5 segments were recorded

root twitches, but this difference is only 1 or $2 \mathrm{~ms}^{22}$ Thus there are limitations to differentiating between afferent, sensory and efferent, motor muscle twitches because measuring such tiny differences, albeit possible, is technically complicated and time-consuming. In our practice, we therefore define the epidural posterior position of the electrode not only based on X-ray studies but also based on neurophysiological studies of motor behavior, analyzing muscle twitches during stimulation with a train of $20-30 \mathrm{~Hz}$. This train yields a stimulus/response ratio of $1: 1$ when applied to anterior roots and a reduced ratio when applied to dorsal roots. ${ }^{23}$ In the present study we have not explicitly addressed these neurophysiological parameters, but the test was performed in all studied subjects. Thus the posterior positions of the cathode were documented by neurophysiological as well as radiological means as part of our routine approach.

Our study stresses the need to focus on the anatomical details of lumbar cord and the discrepant termination points of spinal cord and dural sac. ${ }^{24-26}$ We have demonstrated that by stimulating the posterior median structure of the upper and lower lumbar cord segments at threshold current levels (1$4,5 \mathrm{~V}$ ) we can elicit muscle twitches corresponding to the L2 and L4/L5 cord segments. We should try to avoid stimulating lengthy nerve roots inside the thecal sac while exploring the site of the electrode (see Figure 5). In contrast to its L1/L2 upper segments, the L4/L5 lower segments of the lumbar cord are surrounded by neural structures and dorsal roots of the nerves in the upper segments. This implies that low-amplitude responses in the muscle groups that correspond to upper lumbar segments are presumably due to dorsal root stimulation outside of the lumbar cord area that those dorsal roots actually feed. However, the recruitment order of induced muscle twitches can clarify the site of stimulation since lumbar cord segments are built in the vertical (ie rostral or caudal) direction and the 


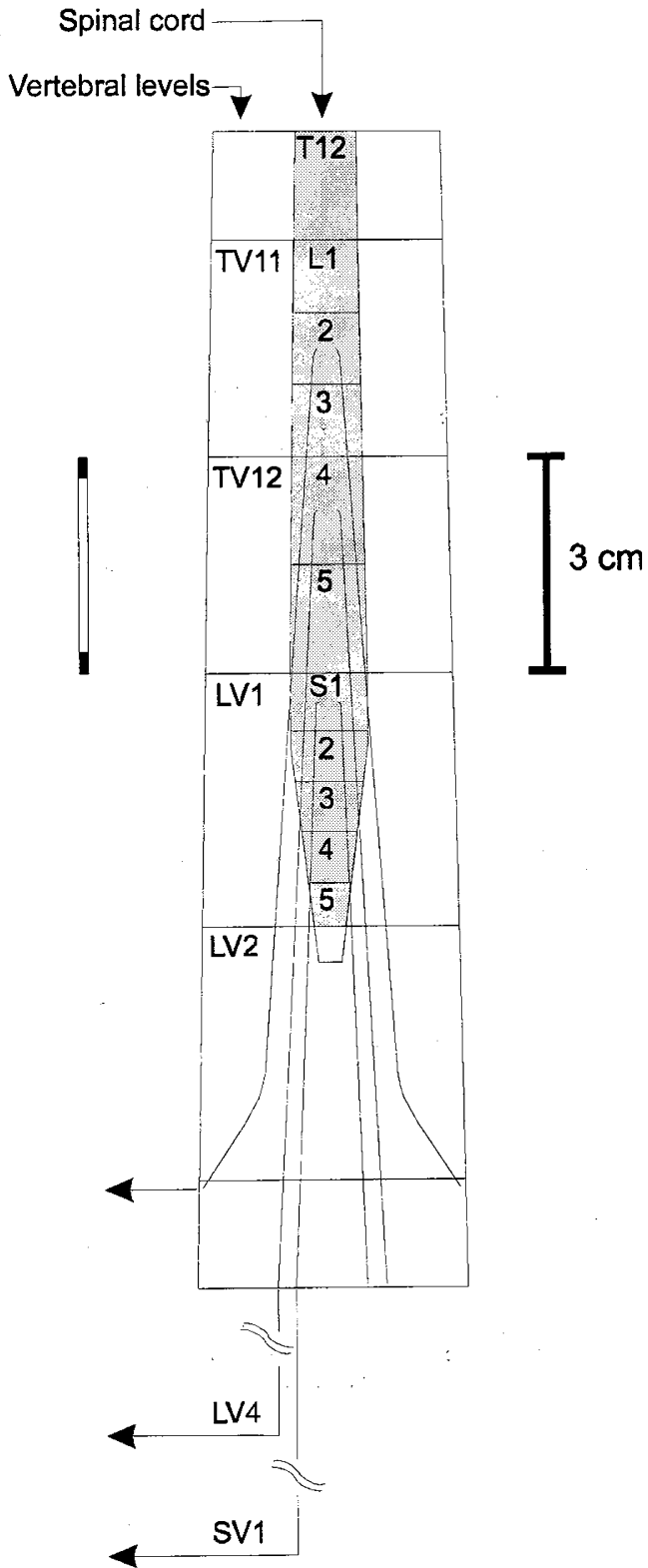

Figure 5 Anatomical sketch of lumbar spine and lumbosacral spinal cord painting relation between vertebras and spinal cord segments and pathway of L2, L4 and S1 roots

distance between lumbar segments are approximately $1.5-2 \mathrm{~cm}$.

Nerve roots have a distinct horizontal layering. Therefore rostral lumbar roots are more lateral, caudal lumbar roots are more medial. ${ }^{19}$ This means that positioning the cathode in a rostral-caudal direction, as compared to a medial-lateral direction, will generate a different recruitment order of recorded EMG of muscle twitches.

Quantitative analysis by computer modeling of the epidural electrical stimulation of posterior structures of the human lumbar $\operatorname{cord}^{27}$ demonstrate actual measurements of relation between stimulus strength, site of stimulation, size and the order of muscle twitches. Thus we have used a functional criteria, in contrast to computer modeling, which is based on computation of electrophysiological parameters of muscle twitches and of dimensions of anatomical structures, electrical properties, electrical field and conducting axons.

To summarize, given the requisite experience it is feasible to identify the posterior roots of the L2 lumbar cord segment. Threshold stimuli applied in the upper lumbar cord segment allow us to record EMG responses of muscle twitches confined to the quadriceps and adductor muscles. When we obtain EMG responses of muscle twitches in the leg, the stimulating electrode is over the L4-L5 cord segments and hence below the target of L2 stimulation.

\section{Acknowledgements}

We wish to thank all patients who participated in this study and the physician who referred them to our program. We are indebted to Ms Auer and Ms Preinfalk for their excellent technical support and to Dr Lamp for sharing this expertise in neurosurgery. Special thanks are due to Ms Alesch for her technical support. We would like to express our gratitude to Prof LE Larsson for his review and valuable suggestions for improvement of manuscript. This work was supported by Ludwig Boltzmann Institute for Restorative Neurology and Neuromodulation in Vienna, Austria and by a grant from Kent Waldrep National Paralysis Foundation in Dallas, Texas, USA.

\section{References}

1 Luc GY. Spinal cord stimulation and chronic critical limb ischemia. Neuromodulation 1999; 2: 1-3.

2 Waltz JM. Chronic Stimulation for Motor Disorders. In: Gildenberg PL, Tasker RR, McGraw Hill (eds). Textbook for Stereotactic and Functional Surgery. New York, 1998, Chapter 113: pp. $1087-1099$.

3 Dimitrijevic MR. Chronic spinal cord stimulation for spasticity. In: Gildenberg PL, Tasker RR, McGraw Hill (eds). Textbook for Stereotactic and Functional Surgery. New York, 1998, 4: Chapter 131: pp. $1267-1273$.

4 Holsheimer J, Barolat G. Spinal geometry and paresthesia coverage spinal cord stimulation. Neuromodulation 1998; 1: $129-136$

5 Struijk JJ, Holsheimer J, Van der Heide GG, Boom HB. Excitation of dorsal root fibers in spinal cord stimulation: a theoretical study. IEEE Trans Biomed Eng 1993; 40: 632-638.

6 Dimitrijevic MR, Faganel J, Sharkey PC, Sherwood AM. Study of sensation and muscle twitch responses to spinal cord stimulation. Int Rehab Med 1980; 2: 76-81.

7 Barolat $\mathrm{G}$ et al. Epidural cord stimulation in the management of spasms in the spinal cord injury: A prospective study. Stereotact Funct Neurosurg 1995; 64: 153-164.

8 Dimitrijevic MR. Motor control in human spinal cord injury. In: Stalberg E, Sharma HS, Olsson Y (eds). Spinal Cord monitoring Springer, Wein: New York, 1998, Chap 16: 409-420.

9 Dimitrijevic MR. Motor control in chronic spinal cord injury patients. Scand J Rehab 1994; 30 (Suppl): 53-62. 
10 Dimitrijevic MR, McKay NB, Sherwood AM. Motor control physiology below spinal cord injury: residual volitional control of motor units in paretic and paralysed muscles in neuronal regeneration, reorganisation and repair. In: Serl FJ (eds). Advanced Neurology. Raven Press: New York, 1997; 72: 335 345.

11 Bohannon RW, Smith AW. Interrater reliability of a modified Ashworth scale of muscle spasticity. Physical Therapy 1987; 67: $206-207$.

12 Frankel HL, Hancock DO, Hyslop G. The value of postura reduction in the initial management of closed injuries of the spine with paraplegia and tetraplegia. Paraplegia 1969; 7: 179-192.

13 Ditunno JF, Young W, Donovan WH, Creasey G. The international standard booklet for neurological and functional classification of spinal cord injury. Paraplegia 1994; 32: 70-80.

14 Carpenter MB. Human Neuroanatomy. Baltimore; Williams \& Wilkins 1976.

15 Struijk JJ, Holsheimer J, Van Veen BK, Boom HB. Epidural spinal cord stimulation: Calculation of field potentials with special reference to dorsal column nerve fibers. Trans Biomed Eng 1991; 38: $104-110$

16 Holsheimer J, Struijk JJ. How do geometric factors influence epidural spinal cord stimulation? A quantitative analysis by computer modeling. Stereotact Funct Neurosurg 1991; 56: $234-$ 249.

17 He J, Barolat G, Struijk J, Holsheimer J. Perception threshold and the electrode position for spinal cord stimulation. Pain 1994; 59: $55-64$.

18 d'Avella D, Mingrino S. Microsurgical anatomy of lumbosacral spinal cord root. J Neurosurg 1979; 51: 819-823.
19 Wall EJ et al. Organization of intrathecal nerve roots at the level of the conus medullaris. J Bone Joint Surg [Am] 1990; 72: 14951499.

20 Wall EJ et al. Cauda equina anatomy I: intrathecal nerve root organization. Spine 1990; 15: $1244-1247$.

21 Cohen MS, Wall EJ, Brown RA. Cauda equina anatomy II, extrathecal nerve root and dorsal root ganglia. Spine 1990; 15: $1248-1251$.

22 Sharkey PC, Dimitrijevic MM, Faganel J. Neurophysiological analyses of factors influencing efficacy of spinal cord stimulation. Appl Neurophysiol 1982; 45: 68-72.

23 Dimitrijevic MR, Faganel J, Sherwood AM. Spinal cord stimulation as a tool for physiological research. Appl Neurophysiol 1983; 46: $245-253$.

24 Baron AJ. The vertebral level of termination of the spinal cord during normal and abnormal development. J Anat 1970; 106: $489-497$

25 Needles JH. The caudal level of termination of the spinal cord in American whites and American Negroes. Anat Rec 1935; 63: $417-424$.

26 Reimann AF, Anson BJ. Vertebral level of termination of the spinal cord with the report of a case of sacral cord. Anat Rec 1944; 88: $127-138$.

27 Rattay F, Minassian K, Dimitrijevic MR. Epidural electrical stimulation of posterior structures of the human lumbosacral cord: 2. Quantitative analysis by computer modeling. Spinal Cord; in submission. 